\title{
External Electric Field-Induced Second-Order Nonlinear Optical Effects in Hexagonal Graphene Quantum Dots
}

Haipeng $\mathrm{Li}^{*}$, Xiaodong Yu, Xiaopeng Shen, Gang Tang, and Kui Han*

School of Physical Science and Technology, China University of Mining and Technology, Xuzhou, P. R. China

Table S1. Band lengths (in $\AA$ ) and band angles (in degree) of optimized hexagonal GQD I under electric fields $\mathrm{F}_{i}$, together with the dipole moment $\mu$ (in $10^{-30} \mathrm{Cm}$ ).

\begin{tabular}{lcccccccccc}
\hline $\mathrm{F}_{x}\left(\times 10^{-4} \mathrm{au}\right)$ & 0 & 50 & 100 & 150 & 200 & 250 & 300 & 350 & 400 \\
\hline $\mathrm{C} 1-\mathrm{C} 2$ & 1.427 & 1.427 & 1.427 & 1.427 & 1.427 & 1.427 & 1.427 & 1.428 & 1.429 \\
$\mathrm{C} 2-\mathrm{C} 3$ & 1.428 & 1.428 & 1.429 & 1.430 & 1.431 & 1.432 & 1.433 & 1.435 & 1.436 \\
$\mathrm{C} 3-\mathrm{C} 4$ & 1.422 & 1.422 & 1.424 & 1.427 & 1.431 & 1.436 & 1.443 & 1.450 & 1.459 \\
$\mathrm{C} 4-\mathrm{C} 5$ & 1.424 & 1.424 & 1.424 & 1.424 & 1.424 & 1.425 & 1.425 & 1.426 & 1.427 \\
$\mathrm{C} 5-\mathrm{H} 1$ & 1.087 & 1.089 & 1.091 & 1.093 & 1.096 & 1.100 & 1.105 & 1.111 & 1.118 \\
$\mu_{x}$ & 0 & 16.11 & 32.43 & 49.24 & 66.83 & 85.67 & 106.58 & 287.79 & 310.44 \\
\hline $\mathrm{F}_{y}\left(\times 10^{-4} \mathrm{au}\right)$ & 0 & 50 & 100 & 150 & 200 & 250 & 300 & 350 & 400 \\
$\mathrm{C} 1-\mathrm{C} 6$ & 1.422 & 1.422 & 1.424 & 1.427 & 1.431 & 1.436 & 1.442 & 1.449 & 1.457 \\
$\mathrm{C} 6-\mathrm{C} 7$ & 1.424 & 1.424 & 1.424 & 1.424 & 1.424 & 1.424 & 1.424 & 1.425 & 1.426 \\
$\mathrm{C} 7-\mathrm{H} 2$ & 1.087 & 1.089 & 1.090 & 1.093 & 1.096 & 1.099 & 1.104 & 1.109 & 1.116 \\
$\mu_{y}$ & 0 & 16.10 & 32.43 & 49.21 & 66.72 & 85.33 & 176.14 & 291.92 & 316.09 \\
\hline $\mathrm{F}_{z}\left(\times 10^{-4} \mathrm{au}\right)$ & 0 & 100 & 200 & 300 & 400 & 500 & 600 & 700 & 750 \\
$\theta(\mathrm{Cl} 1-\mathrm{C2}-\mathrm{C} 3-\mathrm{C} 4)$ & 180 & 178.33 & 176.49 & 174.25 & 171.57 & 168.66 & 177.67 & 179.63 & 179.87 \\
$\theta(\mathrm{C6} 6-\mathrm{Cl}-\mathrm{C2}-\mathrm{C} 3)$ & 180 & -178.35 & -176.54 & -174.33 & -171.62 & -169.43 & -163.75 & -154.69 & -150.77 \\
$\theta(\mathrm{C3} 3-\mathrm{C} 4-\mathrm{C} 5-\mathrm{H} 1)$ & 180 & 178.04 & 176.01 & 173.77 & 171.33 & 168.81 & 169.38 & 170.66 & 171.99 \\
$\mu_{z}$ & 0 & 8.86 & 23.86 & 36.87 & 51.21 & 67.34 & 98.09 & 186.00 & 239.65 \\
\hline & & & & & & &
\end{tabular}


Table S2. Band lengths (in $\AA$ ) and band angles (in degree) of optimized hexagonal GQD II under electric fields $\mathrm{F}_{i}$, together with the dipole moment $\mu$ (in $10^{-30} \mathrm{Cm}$ ).

\begin{tabular}{|c|c|c|c|c|c|c|c|c|}
\hline $\mathrm{F}_{x}\left(\times 10^{-4} \mathrm{au}\right)$ & 0 & 50 & 100 & 150 & 200 & 250 & 300 & 325 \\
\hline $\mathrm{C} 1-\mathrm{C} 2$ & 1.420 & 1.420 & 1.421 & 1.423 & 1.426 & 1.429 & 1.435 & 1.439 \\
\hline $\mathrm{C} 2-\mathrm{C} 3$ & 1.420 & 1.420 & 1.422 & 1.424 & 1.428 & 1.432 & 1.435 & 1.436 \\
\hline C3-C4 & 1.429 & 1.429 & 1.428 & 1.427 & 1.426 & 1.427 & 1.432 & 1.436 \\
\hline $\mathrm{C} 4-\mathrm{C} 5$ & 1.420 & 1.422 & 1.425 & 1.429 & 1.434 & 1.439 & 1.444 & 1.446 \\
\hline C5-C6 & 1.431 & 1.432 & 1.435 & 1.438 & 1.443 & 1.450 & 1.458 & 1.463 \\
\hline C6-C7 & 1.401 & 1.402 & 1.403 & 1.405 & 1.409 & 1.416 & 1.422 & 1.426 \\
\hline C7-H1 & 1.088 & 1.090 & 1.092 & 1.095 & 1.099 & 1.104 & 1.111 & 1.115 \\
\hline$\mu_{x}$ & 0 & 43.89 & 89.43 & 139.06 & 197.91 & 460.46 & 517.17 & 546.38 \\
\hline $\mathrm{F}_{y}\left(\times 10^{-4} \mathrm{au}\right)$ & 0 & 50 & 100 & 150 & 200 & 250 & 300 & 325 \\
\hline $\mathrm{C} 1-\mathrm{C} 8$ & 1.429 & 1.429 & 1.428 & 1.427 & 1.427 & 1.427 & 1.430 & 1.432 \\
\hline $\mathrm{C} 8-\mathrm{C} 9$ & 1.420 & 1.422 & 1.424 & 1.427 & 1.432 & 1.437 & 1.443 & 1.447 \\
\hline C9-C10 & 1.431 & 1.432 & 1.434 & 1.438 & 1.445 & 1.454 & 1.466 & 1.473 \\
\hline $\mathrm{C} 10-\mathrm{C} 11$ & 1.437 & 1.436 & 1.435 & 1.433 & 1.430 & 1.428 & 1.426 & 1.426 \\
\hline $\mathrm{C} 11-\mathrm{H} 2$ & 1.087 & 1.089 & 1.091 & 1.094 & 1.098 & 1.104 & 1.111 & 1.116 \\
\hline$\mu_{y}$ & 0 & 43.90 & 89.30 & 137.96 & 268.09 & 466.11 & 527.49 & 558.93 \\
\hline $\mathrm{F}_{z}\left(\times 10^{-4} \mathrm{au}\right)$ & 0 & 50 & 100 & 200 & 300 & 400 & 450 & 500 \\
\hline$\theta(\mathrm{C} 1-\mathrm{C} 2-\mathrm{C} 3-\mathrm{C} 4)$ & 180 & 179.17 & 178.12 & 176.02 & 172.43 & 165.41 & 159.16 & 157.47 \\
\hline$\theta(\mathrm{C} 8-\mathrm{C} 1-\mathrm{C} 2-\mathrm{C} 3)$ & 180 & -179.18 & -178.19 & -176.04 & -174.01 & -151.76 & -143.16 & -137.74 \\
\hline$\theta(\mathrm{C} 5-\mathrm{C} 6-\mathrm{C} 7-\mathrm{H} 1)$ & 180 & 179.05 & 178.16 & 176.17 & 174.16 & 167.36 & 165.75 & 164.65 \\
\hline$\mu_{z}$ & 0 & 11.50 & 23.13 & 47.69 & 74.88 & 140.59 & 158.62 & 262.86 \\
\hline
\end{tabular}




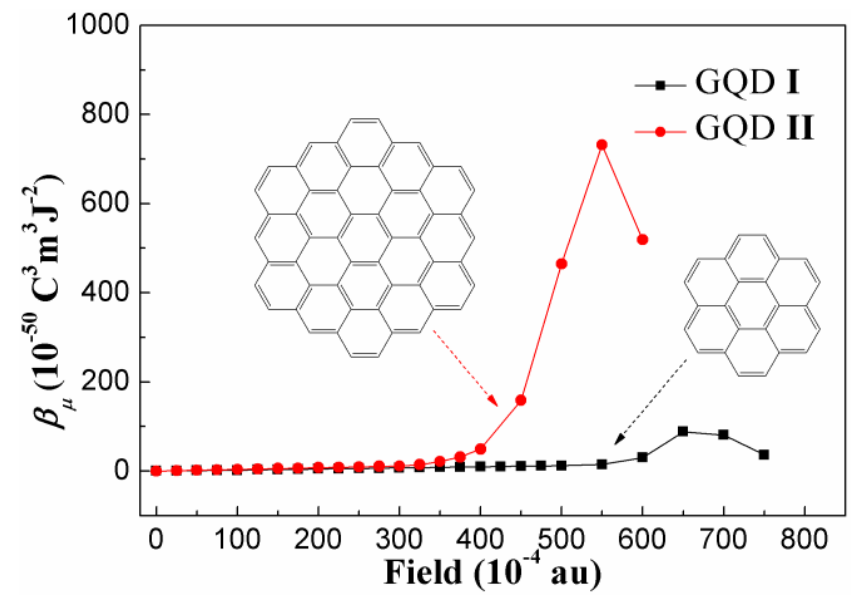

Figure S1. Evolution of the electronic first hyperpolarizability $\beta_{\mu}$ of GQD I and GQD II under electric field $\mathrm{F}_{z}$, respectively.

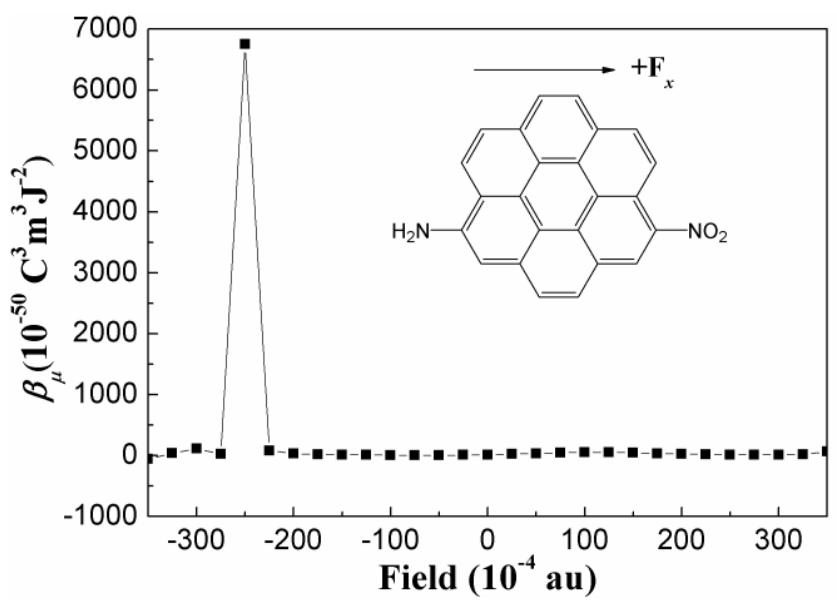

Figure S2. Evolution of the electronic first hyperpolarizability $\beta_{\mu}$ of $\mathrm{NH}_{2}-\mathbf{I}-\mathrm{NO}_{2}$ under electric field $\mathrm{F}_{x}$ ranging from -0.035 au to $0.035 \mathrm{au}$. 